\title{
Mec-associated dru typing in the epidemiological analysis of ST239 MRSA in Malaysia.
}

\begin{abstract}
The usefulness of mec-associated dru typing in the epidemiological analysis of methicillinresistant Staphylococcus aureus (MRSA) isolated in Malaysia was investigated and compared with pulsed-field gel electrophoresis (PFGE), multilocus sequence typing (MLST), and spa and SCCmec typing. The isolates studied included all MRSA types in Malaysia. Multilocus sequence type ST188 and ST1 isolates were highly clonal by all typing methods. However, the dru typing of ST239 isolates produced the clearest discrimination between SCCmec IIIa and III isolates, yielding more subtypes than any other method. Evaluation of the discriminatory power for each method identified dru typing and PFGE as the most discriminatory, with Simpson's index of diversity (SID) values over 89\%, including an isolate which was non-typeable by spa, but dru-typed as dt13j. The discriminatory ability of dru typing, especially with closely related MRSA ST239 strains (e.g., Brazilian and Hungarian), underscores its utility as a tool for the epidemiological investigation of MRSA.
\end{abstract}

Keyword: Dru types; Methicillin-resistant Staphylococcus aureus; Multilocus sequence typing; Epidemiological analysis. 